\title{
Extension and restriction of sleep time in the physical performance of athletes with visual and intellectual disabilities: new possibilities
}

\author{
HELOÍSA P. PANCOTTO'1 | ADRIELLY N. SILVA² | ANDREA M. ESTEVES| \\ ${ }^{1}$ Faculdade de Educação Física, Universidade Estadual de Campinas, Campinas, SP, Brazil. \\ ${ }^{2}$ Faculdade de Ciências Aplicadas, Universidade Estadual de Campinas, Limeira, SP, Brazil.
}

Correspondence to: Andrea Maculano Esteves, R. Pedro Zaccaria, 1300, Limeira - SP, 13484-350.

email: andrea.esteves@fca.unicamp.br

https://doi.org 10.20338/bjmb.v13i4.132

\begin{abstract}
HIGHLIGHTS
- A predominance of the morning chronotype among the athletes;

- Visually impaired athletes experienced poorer sleep quality than intellectually impaired athletes

- Visually impaired athletes presented higher sleep efficiency and lower latencies in the restriction period.
\end{abstract}

$\begin{array}{ll}\text { ABBREVIATIONS } \\ \text { E-types } & \text { evening types } \\ \text { M-types } & \text { morning types } \\ \text { NREM } & \text { non-REM sleep } \\ \text { N-types } & \text { neither types } \\ \text { PSQI } & \text { Pittsburgh Sleep Quality Index } \\ \text { REM } & \text { rapid-eye-movement }\end{array}$

PUBLICATION DATA

Received 08042019

Accepted 23082019

Published 01102019

\begin{abstract}
BACKGROUND: Sleep behavior analysis can provide very useful information on the preparation and performance of athletes in competitions.

AIM: This study aimed to analyze the effect of sleep extension and restriction on the physical performance of athletes with visual and intellectual disabilities.

METHOD: We evaluated 10 athletics athletes who participated in three experimental conditions: basal, extension (+90 $\mathrm{min}$ ), and restriction of sleep (- $90 \mathrm{~min}$ ), through time in bed. Questionnaires on sleep quality (Pittsburgh), sleepiness (Karolinska), and a sleep diary were applied. For performance evaluation, physical tests (simulation of official tests) were performed.

RESULTS: The results demonstrated that blind individuals showed a significant PSQI score for altered sleep quality and an increase in arousal compared to those with an intellectual disability, however, with maintained good sleep efficiency. In addition, although the athletes presented a reduction of $3.52 \%$ in the physical test times at the extension moment and an increase of $0.13 \%$ at the restriction moment, no significant differences were observed.

CONCLUSION: The results present promising data for the analysis of the relationship between sleep extension and reduction in sleep, since a successful response to sleep extension may depend on several factors.
\end{abstract}

\section{INTRODUCTION}

The recommended amount of sleep to achieve optimal health and quality of life varies across the lifespan, with a gradual decrease from birth to older adulthood 1 . According to the American Academy of Sleep Medicine, adults require between 7 and $9 \mathrm{~h}$ of sleep for optimal performance and health, while adolescents require additional sleep, ideally between 8 and $10 \mathrm{~h}^{1}$. The amount of sleep necessary varies widely between individuals and may differ day to day for the same individual based on a number of factors including illness, sleep debt, and physiological or psychological stress ${ }^{2}$.

Studies indicate that people with disabilities may present altered sleep patterns due to factors inherent to their disability ${ }^{3}$. People with visual impairment have a greater number of sleep disturbances, both during rapid-eye-movement (REM) sleep and nonREM (NREM) sleep 3 . Moreover, the absence of visual input to the retina can also induce changes in entrainment of the circadian rhythm, as light is the primary zeitgeber of the master biological clock found in the suprachiasmatic nucleus of the hypothalamus. Blindness is associated with an increased incidence of free-running, or non-24-h, circadian

\begin{tabular}{l|l|l|l|ll}
\hline $\begin{array}{l}\text { Pancotto, Silva, } \\
\text { Esteves }\end{array}$ & 2019 & VOL.13 & N.4 & https://doi.org 10.20338/bjmb.v13i4.132
\end{tabular}


rhythms ${ }^{4}$.

For high performance athletes, frequent exposure to high intensity training programs and competitions in different time zones leads to the need for optimal recovery and, therefore, may increase overall sleep requirement. Actigraphy-based sleep studies reveal that athletes have shown high sleep fragmentation, low sleep efficiency, and, consequently, low sleep durations ${ }^{5}$. This may be important because sleep needs vary among individuals: some may complain of poor sleep quality, although objective indicators indicate sufficient sleep quality, while others report good sleep quality despite objective markers of poor sleep 6 .

Altered sleep patterns such as short, long, irregular, and mistimed sleep are all implicated in a variety of adverse health conditions ${ }^{3}$. However, duration is only one component of sleep. The importance of sleep quality has been increasingly recognized as a vital element of overall health and well-being ${ }^{7}$. Sleep quality includes quantitative aspects of sleep, such as sleep duration, sleep latency, or number of arousals, as well as more purely subjective aspects, such as "depth" or "restfulness" of sleep. However, the exact elements that compose sleep quality, and their relative importance, may vary between individuals ${ }^{8}$.

In this sense, the term "quality" is not used to refer to the amount or distribution of sleep and wakefulness but rather to variations in the experience of sleep itself 7 .

It is generally assumed that high quality and sufficient sleep is necessary for multiple factors that contribute to athletic performance, such as physical and mental recovery from rigorous training regimens, minimizing the risk of injury and avoiding fatigue and lapses in concentration ${ }^{5}$.

Therefore, the purpose of this study was to analyze the effect of sleep extension and restriction on the physical performance of athletes with visual and intellectual disabilities.

\section{METHODS}

This is a cross-sectional research, approved by the Ethics and Research Committee of UNICAMP (2.240.96). Ten athletes with intellectual and visual disability were analyzed in the Training Center in the city of Limeira - SP. All athletes presented an existing functional classification according to the established standards for their disability and modality (athletics), attested by ophthalmologists and psychologists accredited by the Brazilian Paralympic Committee.

For the recruitment of the subjects, the inclusion criteria used were: high performance athletes with visually and intellectual disability, legible in the functional classification of the athletics modality, and participating in national and international competitions of the modality, regardless of age and sex. The athletes with visual impairment had light perception.

Athletes who used continuous medication to control sleep and depression were excluded from the study as well as those who were in a state of depression.

\section{Experimental Design}

After participants had signed the Free and Informed Consent Form agreeing to voluntarily participate in the research, athletes took part in three experimental conditions, 
basal (habitual sleep), sleep extension (+ $90 \mathrm{~min}$ ), and sleep restriction (- $90 \mathrm{~min}$ ) (Figure $1)^{9}$. In the first seven days, the sleep diary was used to determine the standard sleep time and usual total time in bed. Time in bed was calculated from the reported time of lying down and waking up. The average of the 7 days was evaluated. In the second week, the total time in bed was extended by +90 minutes, and in the third week, it was reduced by 90 minutes. The sleep extension and restriction times (90-minutes) were added or reduced at the beginning of bedtime. Participants were instructed to use alarm clocks to advise them when to lie down and get up. In each condition (habitual sleep, extension and restriction of sleep), the individuals completed the sleep diary and on the final day of each period, the athletes performed a physical evaluation (simulation of the main test of each athlete). The Karolinska Sleepiness Scale and Pittsburgh Sleep Quality Index questionnaires were applied at the time of physical evaluation. The chronotype questionnaire was applied only at the first moment.

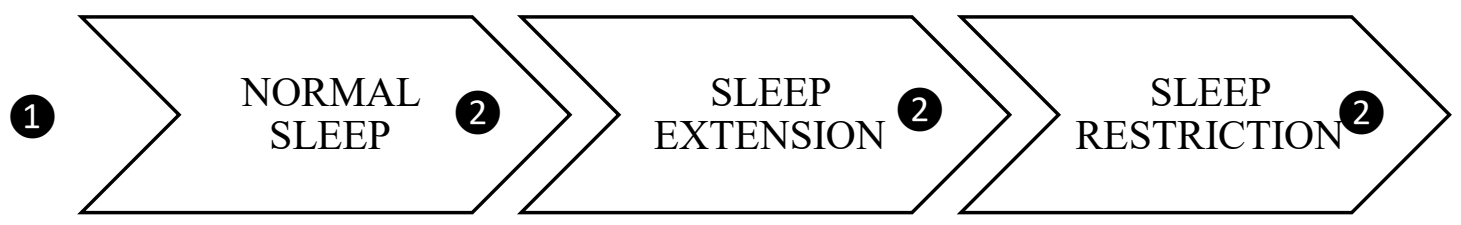

1 Pittsburgh Questionnaire and Horne Östberg Questionnaire.

2 Physical Test, Pittsburgh Questionnaire and Karolinska Scale.

Figure 1. Experimental design.

\section{Experimental Procedure}

Participants were interviewed and evaluated in the facilities of the training center, in a quiet room reserved to answer the questionnaires, which were made available in Braille, in case of preference of the athlete.

Chronotype: To ascertain the chronotype, the subjects answered 19 questions on the questionnaire developed by Horne and Ostberg (1976), previously translated into Portuguese and validated ${ }^{10}$. The sum of the questions provides a score ranging from 16 to 86 points, with lower values corresponding to evening chronotypes ${ }^{11}$.

Pittsburgh Sleep Quality Index (PSQI): consists of 19 questions divided into seven subcategories, including sleep quality, latency, duration, efficiency, disturbance, use of sleep medication, and daytime dysfunction. The sum of the 7 component scores results in the global PSQI score, which may range from 0 to 21 . A lower PSQI score $(<5)$ indicates higher sleep quality. On the other hand, a higher PSQI score (>5) may indicate severe difficulties in all eight components, reflecting poorer sleep quality ${ }^{8}$.

Karolinska Sleepiness Scale: assesses drowsiness at the moment of application, evaluated through the following question: "How are you feeling now? ${ }^{12}$. The scale consists of nine points, on which the value one corresponds to "very alert" and nine "very sleepy". Drowsiness assessments were obtained on the final day (seventh) of each experimental condition (baseline, extension, and restriction) prior to initiating the physical test. 
Sleep diary: designed to evaluate the characteristics of sleep patterns, was completed by the athletes every night, during the three periods of data collection (21 days). The diary includes questions about bedtime, sleeping and waking hours, number of wakefulness episodes at night, quality of sleep, degree of well-being on waking, how to wake up, and the number of naps taken ${ }^{13}$.

\section{Physical Testing}

Physical tests were performed at the end of each period (baseline, extension, and restriction), at the athletes' training center and at the usual training time (morning), respecting the category and specificity related to the test. The performance parameters computed were speed (time) and distance (meters). Thus, the tests were performed according to the test of each athlete, being track or field: 100, 400, and 1500 meters; and weight throwing (4kg and $7 \mathrm{~kg}$ ).

\section{Statistical analysis}

Statistical analysis was performed with the use of a statistical software package (PASW Statistics for Windows, version 18.0, SPSS Inc., Chicago, IL, USA). The Kolmogorov-Smirnov test was used to test for normal distribution. Descriptive statistics were used. For the parametric samples, Factorial ANOVA and Repeated Measures ANOVA with post hoc of Duncan were performed. The Wilcoxon test was performed to compare physical tests in the extension and restriction conditions. For this, a delta $(\Delta)$ of the times in the physical tests was performed using baseline and extension or restriction values. The level of significance was set at $a<0.05$. The data are presented as means \pm standard deviation (SD) and absolute and relative frequency (\%).

\section{RESULTS}

The sample consisted of ten athletics athletes (6 intellectually disabled $-23.5 y$ rs, 1 woman; and 4 visually impaired $-34.33 y r s, 2$ women). The chronotype results (Table 1) demonstrated a predominance of morning type (M-types) chronotypes.

Table 1 - Classification of athletes' chronotype.

\begin{tabular}{lccccc}
\hline Deficiency & $\begin{array}{c}\text { Functional } \\
\text { classification }\end{array}$ & $\begin{array}{c}\text { M-types } \\
\text { Extreme }\end{array}$ & $\begin{array}{c}\text { M-types } \\
\text { Moderate }\end{array}$ & N-types & E-types \\
\hline Visual & T-12 & $75 \%$ & $25 \%$ & - & - \\
Intellectual & T-20 & - & $66.668 \%$ & $16.666 \%$ & $16.666 \%$
\end{tabular}

$\mathrm{T}$ (acronym used for speed and bottom races); T-12: visually impaired T-20: intellectually disabled.

M-types: morning types; N-types: neither types; E-types: evening-types.

In relation to the sleep variables of visually impaired athletes and those with intellectual disability (Table 2), the Pittsburgh questionnaire score presented significantly lower values for athletes with intellectual disabilities $(F(1,24)=12.272$, $p=0.00183$, Factorial ANOVA) in relation to those with visual disabilities. The other variables did not present statistically significant differences. 
Brazilian Journal of Motor Behavior

\section{Research Article}

Table 2 - Comparison between sleep variables of athletes with visual and intellectual disabilities

\begin{tabular}{lcccccc}
\hline \multicolumn{1}{c}{ Sleep Variables } & Basal & $\begin{array}{c}\text { Visual } \\
\text { Extension }\end{array}$ & Restriction & Basal & $\begin{array}{c}\text { Intellectual } \\
\text { Extension }\end{array}$ & Restriction \\
\cline { 2 - 7 } & $46 \pm 25.27$ & $23 \pm 25.27$ & $16 \pm 25.27$ & $20 \pm 4.9$ & $18 \pm 7.14$ & $15 \pm 4.08$ \\
Latency (minutes) - Pittsburgh & $97.98 \pm 16.19$ & $85.38 \pm 5.76$ & $92.22 \pm 7.63$ & $100.3 \pm 5.07$ & $109.4 \pm 6.27$ & $76.6 \pm 14.79^{\#}$ \\
Sleep Efficiency (\%) - Pittsburgh & $7.5 \pm 1.65^{\mathrm{a}}$ & $7.25 \pm 1.43^{\mathrm{c}}$ & $6 \pm 1.63$ & $4.6 \pm 0.61$ & $3 \pm 0.44^{\mathrm{b}, \mathrm{d}}$ & $4 \pm 0.85^{\mathrm{b}}$ \\
$\begin{array}{l}\text { Pittsburgh Sleep Quality Index } \\
\text { (PSQI) }\end{array}$ & & & & & \\
Somnolence - Karolinska Scale & $5 \pm 0.81$ & 5 & $6 \pm 0.57$ & $5.66 \pm 1.33$ & $3.33 \pm 0.61$ & $3.33 \pm 1.20$ \\
Sleep Diary & & & & & & \\
Time went to bed (hours/min) & $21: 53 \pm 19.11$ & $20: 46 \pm 4.50^{\mathrm{a}}$ & $22: 44 \pm 36.5^{\mathrm{b}}$ & $22: 41 \pm 16.3^{\mathrm{b}}$ & $22: 08 \pm 32.26$ & $22: 59 \pm 18.64^{\mathrm{b}}$ \\
Sleep Onset (hours/min) & $22: 17 \pm 19.37$ & $21: 04 \pm 17.0$ & $23: 12 \pm 47.50$ & $22: 57 \pm 20.9$ & $22: 39 \pm 39.89$ & $23: 19 \pm 19.19$ \\
Time woke up (hours/min) & $06: 08 \pm 30.83$ & $06: 46 \pm 4.50$ & $06: 45 \pm 50.50$ & $07: 39 \pm 43$ & $07: 28 \pm 49.58$ & $07: 25 \pm 37.47$ \\
Sleep Offset (hours/min) & $06: 17 \pm 26.52$ & $06: 55 \pm 4.50$ & $06: 53 \pm 53.50$ & $07: 55 \pm 47.3$ & $08: 08 \pm 48.83$ & $07: 40 \pm 41.52$ \\
Bed time (hours/min) & $08: 17 \pm 21.56$ & $09: 23 \pm 25.15$ & $08: 18 \pm 78$ & $09: 06 \pm 35.9$ & $09: 58 \pm 32.46$ & $08: 29 \pm 38.60$ \\
\hline
\end{tabular}

ANOVA FATORIAL, pos hoc Duncan ( $p<0.05)$. a differ $b$; c differ $d ;{ }^{\#}$ extension intellectual $(p=0,06)$.

Reference normal values: Pittsburgh Sleep Quality Index (PSQI) < 5; Latency (minutes)/Pittsburgh < 30 minutes; Sleep Efficiency(\%)/Pittsburgh >85\% (OHAYON et al., 2017, RODRIGUES et al., 2015).

In addition, the results of the sleep diary demonstrated that the visually impaired athletes went to bed earlier in the extension moment in relation to their time restriction moment, as well as compared to the basal and restriction moments of athletes with intellectual disabilities $(F(2,20)=3.8220, p=0.03929$, Factorial ANOVA). Regarding the mean number of awakenings in the 7 days analyzed, the visually impaired athletes demonstrated a significant increase in arousal compared to those with intellectual disability $(F(1$, $\left.{ }_{12}\right)=167.93, p<0.001$, Repeated Measures ANOVA, data not shown).

Table 3 presents the results of the time in the physical tests.

Table 3 - Description of the performance in the physical tests in the basal / extension and basal / restriction moments.

\begin{tabular}{lccc}
\multicolumn{1}{c}{ Physical Tests } & $\begin{array}{c}\text { Disabilities } \\
\text { (athletes' number) }\end{array}$ & Basal/Extension & Basal/Restriction \\
\hline Weight throwing $^{\&}$ & Visual (2) & $5.80 \pm 4.74$ & $1.69 \pm 2.57$ \\
100 meters $^{\$}$ & Visual (1) & $-0.05 \pm 1.50$ & $-0.64 \pm 1.61$ \\
& Intellectual (3) & & \\
400 meters $^{\$}$ & Intellectual (3) & $-6.48 \pm 3.09$ & $0.22 \pm 1.23$ \\
1500 meters $^{\$}$ & Visual (1) & -5.77 & -1.93
\end{tabular}

The performance parameters computed was speed/time (\$) and distance/meters (\&).

Figure 2 shows the comparisons of the deltas $(\Delta)$ in the physical tests between the basal/extension and basal/restriction moments. The findings showed that although the athletes presented a reduction of $3.52 \%$ in the physical test times at the extension moment and an increase of $0.13 \%$ at the restriction moment, the values were not statistically significant (Wilcoxon, $p=0.09$ ). 


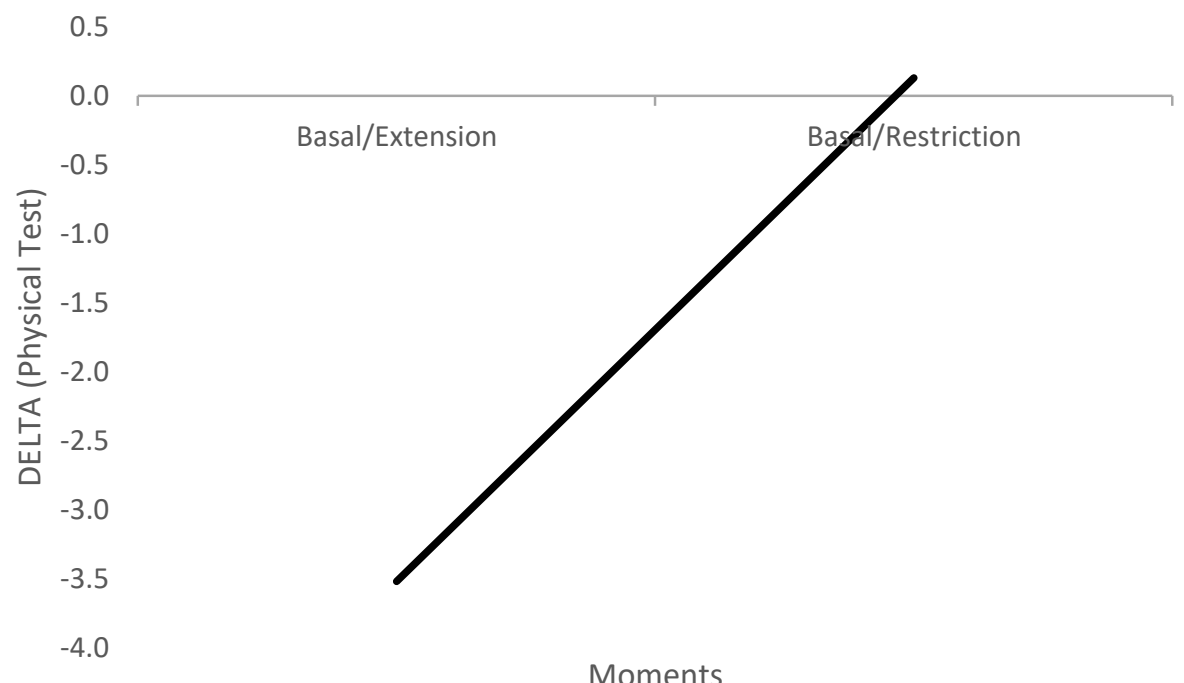

Figure 2. Delta of the times in the physical tests at basal / extension and basal / restriction moments. Wilcoxon, $p=0.09$.

\section{DISCUSSION}

The present study analyzed the effect of sleep extension and restriction, using bed time as a reference, on athletes with visual and intellectual disabilities. The main results demonstrated a predominance of the morning type (M-types) chronotype in the athletes, as well as poor sleep quality in individuals with visual disabilities.

It is important to emphasize that variability in sleep periods is correlated with global PSQI scores. Thus, a higher PSQI score (suggesting lower sleep quality and greater sleep disturbances) is associated, according to Aubin et al., (2016)14, with greater variability in the time of the nocturnal sleep period, both the onset and during sleep. Regular heightened levels of sleep inconsistency are considered unfavorable and thought to disrupt the synchrony of circadian rhythms, subsequently influencing sleep duration and quality ${ }^{15}$. The athletes in the present study with visual impairment presented higher PSQI scores than the population with intellectual disability, showing positive scores for an altered sleep pattern. These results are in agreement with previous studies examining PSQI

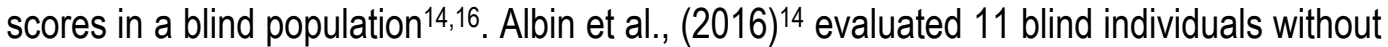
residual light perception and 11 age- and sex-matched normal-sighted controls. The results demonstrated that the majority of blind individuals (7/11) obtained a global PSQI above the clinical threshold for sleep disturbances (PSQI >5), while this was only the case for a minority (3/11) for the sighted controls.

However, the athletes with visual impairment also demonstrated good sleep efficiency (at basal moments and restriction), demonstrating that despite good sleep efficiency, sleep quality was poor. The increase in awakenings may have been one of the factors that influenced the reduction in quality. On the other hand, the athletes with intellectual disability presented good quality of sleep, and the efficiency of the sleep was compatible with the analyzed moments, demonstrating a tendency to lower sleep efficiency 
at the restriction moment. These results suggest that visually impaired athletes may have positive consequences of sleep restriction, since the sleep period is restricted and occurs with better quality.

A study has shown that subjective and objective reports of poor sleep are associated with the circadian phase. O'Donnell et al. (2009)17 showed that sleep quality was perceived as good when synchronized with the normal circadian phase, whereas it was considered weak during conditions of forced desynchrony, that is, when the circadian phase becomes desynchronized with the sleep-wake pattern ${ }^{17}$. In addition, the time and duration of the nocturnal sleep episode also varies with the circadian phase. An increase in the duration of nocturnal sleep is observed when sleep occurs during its typical circadian phase, centered on the melatonin peak and the lower point of body temperature, compared to when sleep occurs outside the circadian phase ${ }^{14}$. In the present study, visually impaired athletes presented light perception, however, studies have shown that alterations in melatonin secretion may be present in this population ${ }^{3,18}$. In addition, all athletes in this study with visual impairment presented morning type (M-type) chronotypes, while the athletes with intellectual disabilities presented characteristics of neither types ( $N$ types) or evening types (E-types).

The performance test results showed that athletes did not present a difference in performance between the moments of extension and restriction of sleep (non-significant data, $p=0.09$ ). Although sleep extension studies have begun to examine the relationship between obtaining extra sleep and cognitive functioning, another line of research has been investigating the effects of prolonged sleep for relatively longer periods of time on physical performance ${ }^{19}$. Studies have shown that the extent of sleep can have a positive impact on athlete well-being and performance ${ }^{20,21}$.

The data of the present study are preliminary, and, thus, the sample was reduced, which may have influenced the responses to the presented protocols. A possible limitation of the study is the lack of direct control of the time programmed to sleep and wake up, as the athletes were simply asked to use an alarm clock. In addition, since the athletes were selected from different modalities, with specific measures (e.g., strength and speed), they may present different relationships with sleep quality.

However, the initial results present promising data for the analysis of the relationship between sleep extension and reduction in sleep, since the successful response to sleep extension may depend on several factors, including individual sleep requirements, load and intensity of training, duration of sleep extension, and the quality of usual sleep before the extension of sleep.

\section{CONCLUSION}

In conclusion, the present study demonstrated that although no relationship was found between the extent and restriction of sleep and performance, the blind athletes presented a significant PSQI score for altered sleep quality, although demonstrating good sleep efficiency. Thus, we demonstrated that despite good habitual sleep efficiency, the athletes did not recover well from other components that can lead to good sleep quality, such as latency of sleep, subjective sleep quality, sleep disturbances, use of sleeping medications, and daytime dysfunction. 


\section{REFERENCES}

1. Paruthi S, Brooks LJ, D'Ambrosio C, Hall WA, Kotagal S, Lloyd RM, Wise, MS. Consensus Statement of the American Academy of Sleep Medicine on the Recommended Amount of Sleep for Healthy Children: Methodology and Discussion. Journal of clinical sleep medicine: JCSM: official publication of the American Academy of Sleep Medicine, 2016; 12(11): 1549-1561.

2. Kirschen GW, Jason JJ, Lauren $\mathrm{H}$. The Impact of Sleep Duration on Performance Among Competitive Athletes: A Systematic Literature Review. Clin. J. Sport Med. 2018; 0:1-10.

3. Aubin S, Christensen, JAE, Jennum, P, Nielsen T, Kupers R, Ptito M. Preserved sleep microstructure in blind individuals. Sleep Medicine. 2018; 42 :21-30.

4. Flynn-Evans EE, Tabandeh H, Skene DJ, Lockley SW. Circadian rhythm disorders and melatonin production in 127 blind women with and without light perception. J. Biol. Rhythms, 2014; 29: 215-224.

5. Lastella M, Roach GD, Halson SL, Sargent C. Sleep/wake behaviours of elite athletes from individual and team sports. Eur. J. Sport Sci. 2015; 15: 94-100.

6. Krystal AD,Edinger JD. Measuring sleep quality. Sleep Med. 2008; 9 (1):10-7.

7. Bird SP. Sleep, recovery, and athletic performance: a brief review and recommendations. Strength Cond. J, 2013; 35:43Y7.

8. Buysse DJ, Reynolds CF, Monk TH, Berman SR, Kupfer DJ. The Pittsburgh Sleep Quality Index: A new instrument for psychiatric practice and research. Psychiatry Research, 1988; 28 (2):193-213.

9. Arnal PJ, Lapole T, Erblang M, Guillard M, Bourrilhon C, Léger D, Chennaoui M; Millet GY. Sleep Extension before Sleep Loss: Effects on Performance and Neuromuscular Function. Med Sci Sports Exerc. 2016;48(8):1595-603.

10. Benedito-Silva AA, Menna-Barreto LS, Marques N, Tenreiro S. A self-assessment questionnaire for the determination of morningnesseveningness types in Brazil. In: HAYES, D. K.; PAULY, J. E.; REITER R. J.; (Org.). Chronobiology: its role in clinical medicine, general biology and agriculture, part B. New York: Wiley-Liss. 1990.

11. Sack RL, Auckley D, Auger R, Carskadon MA, Wright KP, Vitiello MV, Zhdanova IV. Circadian rhythm sleep disorders. 1. Basic principles, shift work and jet lag disorders. An American Academy of Sleep Medicine review. Sleep. 2007.

12. Akerstedt T, Gillberg M. Subjective and objective sleepiness in the active individual. International Journal of Neuroscience, 1990; 52: 29-37.

13. Mantua J, Gravel N, Spencer, RM. Reliability of Sleep Measures from Four Personal Health Monitoring Devices Compared to Research-Based Actigraphy and Polysomnography. Sensors (Basel, Switzerland), 2016; 16(5), 646.

14. Aubin $S$, Gacon $C$, Jennum P, et al. Altered sleep-wake patterns in blindness: a combined actigraphy and psychometric study. Sleep Med, 2016; 24:100-108. 
Brazilian Journal of Motor Behavior

15. Caia J, Halson SL, Scott TJ, Kelly VG. Intra-individual variability in the sleep of senior and junior rugby league athletes during the competitive season. Chronobiol Int. 2017;34(9):1239-1247.

16. Meaidi A, Jennum $P$, Ptito $M$, et al. The sensory construction of dreams and nightmare frequency in congenitally blind and late blind participants. Sleep Med, 2014; 15 (5): 586595.

17. O'Donnell D, Silva EJ, Muench M. et al. Comparison of subjective and objective assessments of sleep in healthy older subjects without sleep complaints J Sleep Res, 2009; 18 (2): 254-263.

18. Leger D, Guilleminault C, Defrance R, et al. Prevalence of sleep/wake disorders in persons with blindness ClinSci, 1999; 97: 193-199.

19. Mah CD, Mah KE, Kezirian EJ, Dement WC. The effects of sleep extension on the athletic performance of collegiate basketball players. Sleep, 2011; 34, 943-950.

20. Swinbourne R, Miller J, Smart D, et. al. The Effects of Sleep Extension on Sleep, Performance, Immunity and Physical Stress in Rugby Players. Sports, 2018; 6 (2), 42.

21. Van Ryswyk E, Weeks R, Bandick L, O'Keefe M, Vakulin L, Catchside P, Barger L, Potter $A$, et al. A novel sleep optimisationprogramme to improve athlete's well-being and performance. Eur. J. Sport Sci. 2017; 17, 144-151.

\section{ACKNOWLEDGEMENTS}

We would like to thank Clube Inclusivo (Limeira/SP) and Coordenação de Aperfeiçoamento de Pessoal de Nível Superior- Brasil (CAPES) - Código de Financiamento 001.

Citation: Pancotto HP, Silva AN, Esteves AM. Extension and restriction of sleep time in the physical performance of athletes with visual and intellectual disabilities: new possibilities. BJMB.2019:13(4): 104-112.

Editor: Dr Fabio Augusto Barbieri - São Paulo State University (UNESP), Bauru, SP, Brazil; Dr José Angelo Barela -

São Paulo State University (UNESP), Rio Claro, SP, Brazil; Dr Natalia Madalena Rinaldi - Federal University of Espírito Santo (UFES), Vitória, ES, Brazil.

Copyright: @ 2019Pancotto, Silva and Esteves and BJMB. This is an open-access article distributed under the terms of the Creative Commons Attribution-NonCommercial-NoDerivatives 4.0 International License which permits unrestricted use, distribution, and reproduction in any medium, provided the original author and source are credited.

Funding: There was no funding for this study.

Competing interests: The authors have declared that no competing interests exist.

DOI: https://doi.org 10.20338/bjmb.v13i4.132 